\title{
Generation of synchronized trains of picosecond laser pulses at two wavelengths in a single-cavity synchronously mode-locked dye laser
}

\author{
O. Buccafusca, J. J. Rocca, M. C. Marconi, and C. S. Menoni \\ National Science Foundation Center for Optoelectronic Computing Systems, and Department of Electrical \\ Engineering, Colorado State University, Fort Collins, Colorado 80523
}

(Received 6 April 1992; accepted for publication 11 September 1992)

\begin{abstract}
Synchronized trains of 5 ps pulses at two wavelengths were generated in a single-cavity synchronously pumped dye laser containing a mixture of two dyes in a single jet and intracavity spatial masking for frequency selection. The utilization of this dual wavelength dye laser to monitor the relaxation of photoexcited carriers in multiple quantum wells was demonstrated.
\end{abstract}

Synchronized trains of picosecond laser pulses of two different wavelengths constitute a powerful tool to study fast processes in materials. In the last several years this scheme was used to study vibrational dephasing of molecular monolayers on surfaces, ${ }^{1}$ optical nonlinearities in the polydiacetylene PTS conjugated polymer, ${ }^{2,3}$ and the solvent dependance of the rotational diffusion of Cresyl Violet, ${ }^{4}$ among other phenomena. To generate the synchronized pulse trains utilized in these studies, two separate dye lasers were pumped in parallel by the same pump laser.

Alternatively, an electro-optic modulator has been introduced in a single synchronously mode-locked dye laser to obtain dual wavelength operation by interleaving the pulse train amplification in the dye medium. ${ }^{5}$ The generation of synchronized picosecond pulses at two wavelengths has also been demonstrated using two cavities that share a common gain medium in a synchronously mode-locked continuous wave dye laser. ${ }^{6,7}$ Pulses of 5 ps of duration were obtained in an arrangement in which each cavity had an overall length twice that of the argon ion pump laser to give sufficient separation between the beams to allow two mirrors to be mounted in separate translation stages. The average jitter between pulses of different wavelength was 20 ps. $^{6}$ In a different dual-cavity arrangement, passive mode locking of one cavity acted as a gain modulator for the second one to generate two independently tunable wavelengths. ${ }^{7}$ In another approach a dye laser having a Rhodamine $6 \mathrm{G}$ and a Cresyl Violet jet introduced in the same cavity produced $4-5 \mathrm{ps}$ pulses at two tunable wavelengths. ${ }^{8}$ In this scheme the laser beam produced by pumping the Rhodamine jet with a mode locked argon ion laser pumped a second jet containing Cresyl Violet.

Herein we report the simultaneous generation of picosecond pulses at two different wavelengths from a single dye laser cavity of the type commonly used to generate femtosecond pulses at a single wavelength by hybrid mode locking. Tunable dual wavelength operation was achieved mixing two dyes in a single jet to increase the bandwidth of the gain medium and utilizing two intracavity prisms to compensate for the difference in transit time of the pulses corresponding to the two different wavelengths. Selection of the laser frequencies was obtained by means of an intracavity spacial filter. By making use of a laser configuration available in many laboratories, this dual wavelength laser scheme has advantages of simplicity and improved pulse synchronization associated with a single cavity.
The dye laser consists of a dual-jet linear cavity arrangement, ${ }^{9}$ which includes a pair of interactivity prisms for group velocity dispersion compensation. For dualwavelength operation, the absorbent jet was turned off and the gain medium was prepared mixing Rhodamine 590, $1.4 \times 10^{-3} \mathrm{M}$, and $\mathrm{DCM}, 3.1 \times 10^{-3} \mathrm{M}$, in a solvent composed of $68 \%$ ethylene glycol and $32 \%$ benzene alcohol. A small amount of Kiton Red, $0.2 \times 10^{-3} \mathrm{M}$, was also added to assist in dissolving the DCM. The dye laser was pumped by a frequency-doubled mode-locked cw Nd-YLF laser with an average power of $850 \mathrm{~mW}$ at $527 \mathrm{~nm}$ and a pulse duration of $40 \mathrm{ps}$ at a repetition rate of $76 \mathrm{MHz}$. The absorption of DCM is negligible at wavelengths above 595 $\mathrm{nm}$, the lowest wavelength produced by the Rhodamine dye, consequently the pumping of both dyes is almost exclusively due to the $527 \mathrm{~nm}$ radiation from the frequencydoubled Nd:YLF laser.

Wavelength selection was achieved by introducing a spatial mask at the high reflectivity end of the cavity, where the wavelengths are dispersed by the prisms. At this location a gold wire $12 \mu \mathrm{m}$ diameter and two lateral metallic blades were positioned for wavelength selection. Synchronized pulses of less than $5 \mathrm{ps}$ of duration in the orange and in the red, separated in wavelength by approximately $30 \mathrm{~nm}$, were obtained. The alignment and cavity length were adjusted in order to obtain similar pulsewidths at both wavelengths. A spectrum of the output beam of the dual wavelength laser is shown in Fig. 1. The pulses centered at $607 \mathrm{~nm}$ had a bandwidth of $4.6 \mathrm{~nm}$ and an average power of $24 \mathrm{~mW}$, while the pulses centered at $640 \mathrm{~nm}$ had a bandwidth of $9.2 \mathrm{~nm}$ and an average power of $13 \mathrm{~mW}$. It was possible to adjust the relative power of the two wavelength components by varying the dye concentrations.

Figures 2(a) and 2(b) show the autocorrelation traces of the pulses corresponding to each wavelength. The autocorrelation traces were generated one at the time by adjusting the angle of a $\mathrm{LIO}_{3}$ crystal to generate the second harmonic of each wavelength component. Tunability with $10 \mathrm{~nm}$ was readily achieved for both pulse trains while maintaining the pulse widths below 6 ps. No effort was made to extend the tunability beyond this range, as it was not required by our particular application.

Figure 2(c) shows a cross-correlation signal of 5 ps pulse trains at 603 and $630 \mathrm{~nm}$. The cross-correlation profile was generated by monitoring the variation of the sum frequency signal in a KDP crystal, as the relative delay of 


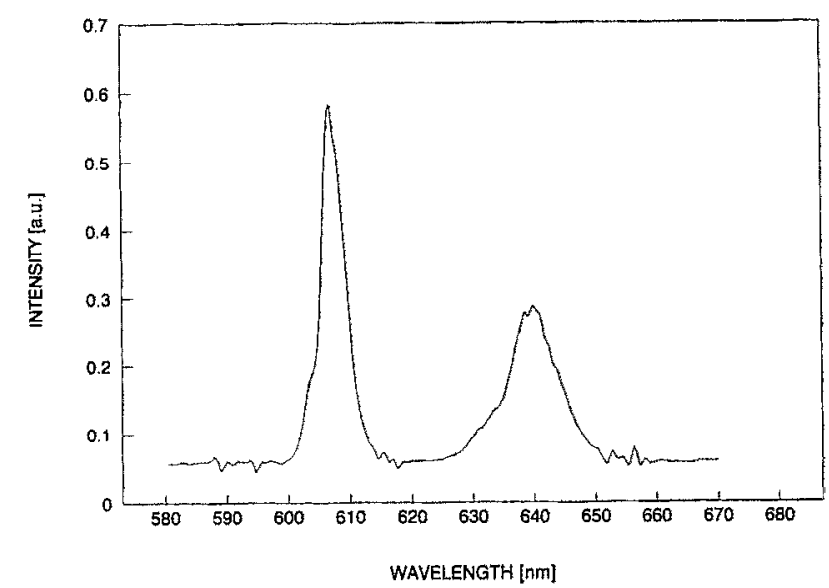

FIG. 1. Spectrum of the dual-wavelength lascr. The pulse train centered at $607 \mathrm{~nm}$ had an average output power of $24 \mathrm{~mW}$, while the pulse train centered at $640 \mathrm{~nm}$ had $13 \mathrm{~mW}$. The pulse width was $5 \mathrm{ps}$ at both wavelengths.

the pulse trains was changed. From the cross-correlation profile, and taking into account the pulse width determined by the autocorrelation measurements, the jitter between the orange and red pulses is estimated to be less than 0.5 ps.

This dual-wavelength laser was used in pump and probe absorption measurements on InGaP/InAlP multiple quantum well (MQW) structure. The MQW was grown by gas source $\mathrm{MBE}$ and consists of 30 periods of $8.4 \mathrm{~nm}$ InGaP wells separated by $22 \mathrm{~nm}$ InAlP barriers. ${ }^{10}$ For these measurements the laser pulses were separated by an available dichroic beamsplitter into two beams having orange/red power ratios of $6 / 1$ and $1 / 10$, respectively. Filters were used to further improve these intensity ratios to $30 / 1$ and more than $1 / 100$, respectively. Alternatively the pulse trains could be separated by placing the output cou-

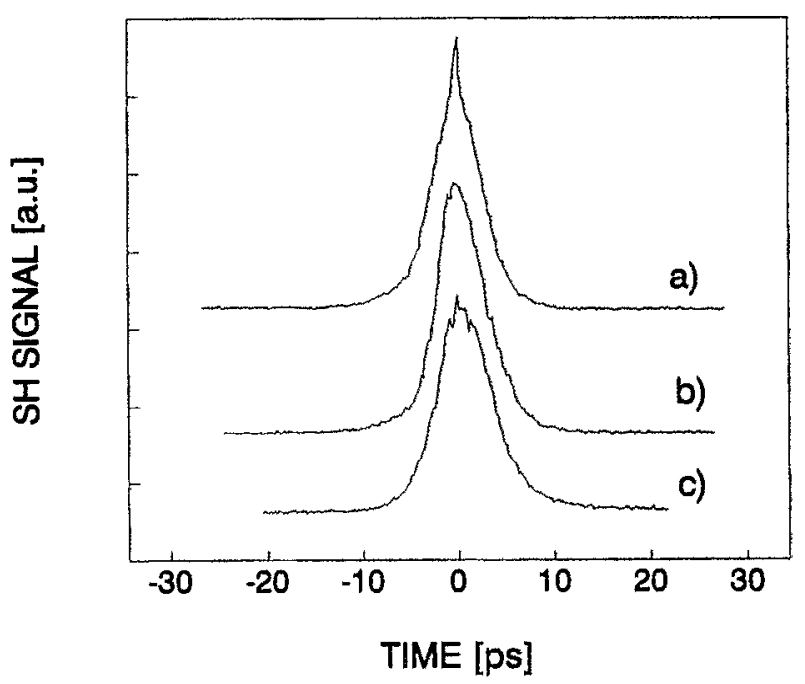

FIG. 2. Autocorrelation signal of the orange (a) and the red (b) pulses obtained by second harmonic generation (SHG) in $\mathrm{LiIO}_{3} 1 \mathrm{~mm}$ thick. Pulse widths are calculated to be 5 ps assuming hyperbolic secant squared pulse profiles. Cross-correlation signal (c) obtained by sum frequency generation in KDP using a pump and probe setup, obtained without lock-in techniques.

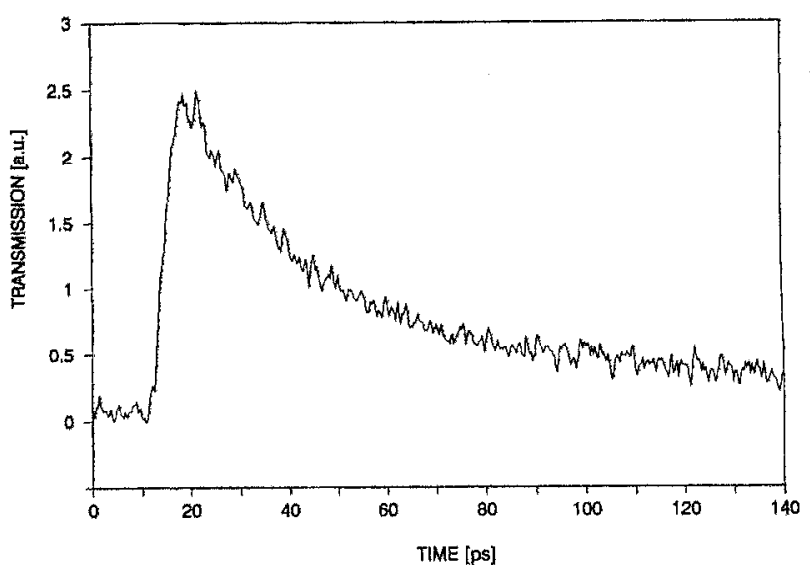

FIG. 3. Change in transmission of an InGaP/InAlP multiple quantum well structure at $630 \mathrm{~nm}$, when pumped by $603 \mathrm{~nm}$ pulses, as a function of the delay between the pulses. The MQW structure was composed by 30 periods of $8.4 \mathrm{~nm}$ InGaP wells separated by $22 \mathrm{~nm}$ InAlP barriers. The transmission recovery time constant is 34 ps.

pler at the end of the cavity adjacent to the prism pair and spatial filter. The pulses of shorter wavelength were used to excite carriers in the sample while the longer wavelength pulses, attenuated to be five times weaker than the pump, were used to probe the changes in the absorption at a lower energy in the conduction band due to the thermalization of carriers to the lattice temperature. Figure 3 shows the absorption recovery of the MQW at $630 \mathrm{~nm}$ after being excited by pulses centered at $603 \mathrm{~nm}$. The measured absorption recovery time was 34 ps.

In summary, we have generated synchronized trains of picosecond pulses at two different wavelengths from a single cavity dye laser. The use of a single cavity allows for low jitter between the pulse trains. Pulses shorter than 5 ps were obtained in the orange and in the red, separated by approximately $30 \mathrm{~nm}$. Tunability of about $10 \mathrm{~nm}$ around 609 and $635 \mathrm{~nm}$ was possible keeping the pulse widths below 6 ps. Pump and probe absorption measurements in InGaP/InAlP MQW has shown the applicability of this laser to the study of fast phenomena in materials.

We thank $M$. Hafich and $G$. Robinson for supplying the MQW sample, and $\mathrm{O}$. Martinez for fruitful discussions. This work was supported by the NSF Center for Optoelectronic Computer Systems through NSF Grant No. ECD 9015128, and by the Colorado Advance Technology Institute. C. S. Menoni was supported by the Engineering Foundation AFOSR Research Initiation Grant No. RI-B91-16.

'J. P. Heritage, Appl. Phys. Lett. 34, 470 (1979).

${ }^{2}$ G. J. Blanchard, J. P. Heritage, A, C, Von Lehmen, M. K. Kelly, G. L. Baker, and S. Ethemad, Phys. Rev, Lett. 63, 887 (1989).

:G. J. Blanchard and J, P. Heritage, J. Chem. Phys. 93, 4377 (1990).

${ }^{4}$ G. J. Blanchard, J. Chem. Phys. 87, 6802 (1987).

${ }^{5}$ S. Blit, A. Olson, and C. L. Tang; Opt. Lett. 4, 245 (1979).

${ }^{6}$ T. F. Lillico, I. S. Rudock, and R. Illingworth, Opt. Commun. 56, 354 (1986).

${ }^{7}$ B. Couilland and A. Ducasse, Appl. Phys. Lett. 29, 665 (1976).

${ }^{\star}$ H. Mahr, IEEE J. Quantum Electron, QE 12, 554 (1979).

${ }^{4}$ M. D. Dawson, T. F. Boggess, D. W. Garvey, and A. L. Smirl, Opt. Commun. 60, 79 (1986).

${ }^{10} \mathrm{M}$. J. Hafich, H. Y. Lee, G. Y.Robinson, D. Li, and N. Otsuka, J. Appl. Phys. 69, 752 (1991). 